\title{
The neurodevelopmental basis of schizophrenia: clinical clues from craniofacial dysmorphology in northwest Ethiopia, 2020
}

Binalfew Tsehay ${ }^{1}$ and Girma Seyoum²*

\begin{abstract}
Background: The neurodevelopmental speculation of schizophrenia states that the pathogenesis of schizophrenia starts with early fetal or neonatal neurocraniofacial development rather than youthful adulthood when manic signs and symptoms are evident. However, there is no direct evidence of a pre-or peri-natal lesion associated with schizophrenia, rather indirect evidence of impaired development can be seen in macroscopic anatomical variations as well as microscopic immunohistochemical anomalies. One approach to studying neurodevelopmental disturbances among schizophrenic patients is somatic physical evidence or neurodevelopmental markers. Thus Our study aimed to assess the neurodevelopmental basis of schizophrenia clinical clues from anthropometric assessment of craniofacial dysmorphology among schizophrenic patients in North West Ethiopia 2019-2020.

Method: Institutional-based comparative cross-sectional study design was conducted in Debre Markos comprehensive specialized hospitals in 190 schizophrenic patients, 190 1st-degree relatives, and 190 healthy controls. Data were collected using standard methods, entered into EpiData version 3.1, and exports to SPSS version 24 for analysis. Descriptive data were analyzed using descriptive statistics. Welch ANOVA and post hoc comparison, a Games-Howell test, were conducted. Significance was set at a $p$-value of $a=0.05$. Read back analysis was also conducted for the conclusion.
\end{abstract}

Results: Five hundred seventy study samples, male 375(65.8\%), and female 195 (34.2\%), were included in this study. The Games-Howell test revealed that the coronal arc length and sagittal arc length among schizophrenic patients were statistically significantly longer than the healthy controls $(p<0.006 ; p<0.001$, respectively). However, the difference between schizophrenic and healthy control regarding head circumference was marginally significant $(p=0.056)$. Schizophrenic patients had a significantly shorter total facial height $(p<0.001)$ and upper facial height $(p<0.001)$ than healthy controls. Regarding facial depth, schizophrenic patients had significantly shallow upper facial depth $(p<0.001)$, middle facial depth $(p=0.046)$, and lower facial depth $(p<0.001)$.

Conclusion: our finding indicated indirect evidence for disturbed craniofacial development in schizophrenia patients, and close and read back analysis of the result supported the neurodevelopmental basis of disease.

Keywords: Craniofacial dysmorphology, Schizophrenia, Neurodevelopment, Ethiopia

*Correspondence: girma91@yahoo.com

2 Department of Anatomy, Addis Ababa University, Addis Ababa, Ethiopia

Full list of author information is available at the end of the article

\section{Introduction \\ Background}

The etiology and pathophysiology of schizophrenia remain poorly understood even though the availability of advanced diagnostic modalities. The neurodevelopmental 
theory of schizophrenia, the widely accepted theory, states that the pathogenesis of schizophrenia starts with early intrauterine fetal or neonatal neurodevelopment rather than during adulthood when manic signs become apparent [1-4]. The developmental disturbances during early life specifically during the period of embryogenesis underlie the structural and functional changes in the brain and the craniofacial region $[5,6]$.

The researchers on the various neurodevelopmental theories believe that the primary pathogenic defect of schizophrenia occurred during the early neurocraniofacial development with an essentially static process of the causative agents. The behavioral consequences of the patients i.e., manic signs and symptoms remain relatively latent until puberty [7].

Generally, the craniofacial region and the brain develop from the same ectodermal tissue during the 5-13th weeks of gestation $[5,8,9]$. If a disturbance occurs during this time, the process of dysmorphology may leave "fossil marks" in the craniofacial regions, which are clinically dormant until after puberty, and the corresponding parts of the brain. According to the neurodevelopmental theory of schizophrenia, neurodevelopmental disorders are often concentrated in the craniofacial region of schizophrenic patients $[5,10,11]$.

If schizophrenia is a neurodevelopmental disorder, individuals with schizophrenia should show an increased rate of craniofacial dysmorphic features which are clinically and cosmetically insignificant [12-14]. However these features can be used as markers of disturbed development or clues in the diagnosis of a specific pattern of disease; may provide indirect clues to brain pathology, and could provide clues to the process of schizophrenia as they reflect adverse events during critical periods of development [15].

There is no direct evidence of a pre-or peri-natal lesion associated with schizophrenia, but indirect evidence of impaired development can be seen in macroscopic anatomical variations as well as microscopic immunohistochemical anomalies. Somatic physical evidence or neurodevelopmental markers, measurable in adults, may reflect abnormal neurodevelopmental processes that occurred before or shortly after birth [16, 17]. Craniofacial dysmorphology may provide another clue to the neurodevelopmental processes in schizophrenia since cerebral morphogenesis is very closely related to craniofacial morphogenesis and is shaped by shared morphogenetic forces $[18,19]$.
Embryologically inferred measures of craniofacial dysmorphology in psychiatric diseases like schizophrenia may give an understanding of their fundamental etiologies and pathogenesis among patients in Ethiopia. Besides, based on destiny outline correspondences, the pattern of anomalies inside embryonic primordia may predict loci of brain maldevelopment, which can be assessed in brain imaging studies [20]. An early Assessment of craniofacial dysmorphology in the population could be used as a tool among diagnostic criteria in making an early screening of schizophrenia.

\section{Methods \\ Study design and setting}

The institutional-based comparative cross-sectional study design was conducted among 190 schizophrenic patients, 190 1st-degree relatives, and 190 healthy controls from December 2019 to June 2020 in Debre Markos referral hospital, Debre Markos. Debre Markos referral hospital and Debre Markos university host a psychiatry center in Debre Markos town that serves the East Gojjam, West Gojjam, and Awi zones.

\section{Study participants}

There were three study groups: The patient group with schizophrenia, the healthy control group who did not have a personal or family history of psychosis and who did not have a current mental illness, first-degree relatives of the patient groups (siblings and parents of patients) in the study and had no schizophrenia themselves.

Selection criteria: Schizophrenic patients and their 1st-degree relatives were recruited from Debre Markos comprehensive specialized hospital psychiatry outpatient department during study periods. The healthy controls were selected from Debre Markos comprehensive specialized hospital and Debre Markos university medical school staffs and students. Exclusion criteria for all study groups included a history of alcoholism or drug addiction, a history of somatic disorder with neurological components, history of head injury, epilepsy, identified craniofacial syndrome, diagnosed organic brain disease, oral-maxillofacial surgery, and schizophrenic patients with aggressive and impulsive behaviors. First-degree relatives with schizoaffective disorder and who met DSMIII-R diagnostic criteria for a psychotic disorder were excluded from the study. Controls were also excluded if 
they had a first-degree relative with a history of psychosis and psychiatric hospitalization.

\section{Sample size calculation}

The sample size was calculated using Fleiss CC Formula on epi info version 7.0 software; by setting a significance level of $95 \%$, power of $80 \%$, and a case-control ratio of 1:1. The final sample size was 570 (190 Cases and 190 Controls, and 190 1st degree relatives.

\section{Data collection procedures and quality control}

Sociodemographic and psychiatric characteristics of the study sample were collected using a structured interviewer-administered questionnaire. The diagnosis of patient control status was based on the Statistical Manual of Mental Disorders 3rd edition (DSM-III-R) by senior psychiatrists and mental health professionals.

Assessment of craniofacial dysmorphology: anthropometric assessment of craniofacial dysmorphology, measurement tools, positioning of the subject, anthropometric landmarks, and methods of measurement were based on Farkas craniofacial atlas, Anthropometry of the Head, and Face [21]. Craniofacial dimensions were measured unilaterally and bilaterally among schizophrenic patients, their 1st-degree relatives, and healthy control using a digital caliper and tape meter to the nearest $0.1 \mathrm{~cm}$, as distances between standard anthropological landmarks. Standard or rest positions of the head are employed, depending on the requirements for each measurement. Some distance measurements and all inclinations are recorded with the subject's head in the Frankfort horizontal position. An anthropometric assessment of craniofacial dysmorphology was performed by well-trained assessors. The training was given by senior anatomists specifically regarding anthropometric landmarks based on Farkas craniofacial atlas, Anthropometry of the Head, and Face. The assessors were blind to patient/control status throughout the evaluations. Many landmarks lie directly on the craniofacial surface, and others are identified by their underlying bony structure. (Additional file 1). The following craniofacial measurements were taken from the cranial region: Head length (glabella to opsithocranion); head width (skull base width) (between both tragions); head height (Frankfurt horizontal plane to the vertex); axial arc length (Head circumference); coronal arc length (arc length between both tragions through the vertex); sagittal arc length (arc length between glabella and opsithocranion, through the vertex).
From the facial region: total facial height (from the trichion to the gnathion); the upper facial height (the trichion to the subnasale); trichion to glabella;glabellasubnasale;glabella-stomion;glabella-gnathion;gnathionnasion, the lower facial height (the subnasale to the gnathion), tragus to subnasale (middle facial depth), tragus to gnathion (lower facial depth), tragus to trichion (upper facial depth) and intercanthal distance (distance between tear ducts) were assessed.

\section{Variables of the study}

The dependent variables were craniofacial dysmorphology among study subjects, whereas sociodemographic characteristics and patient control status were independent variables.

\section{Definition of variable \\ Schizophrenia}

Schizophrenia is a severe mental disorder, diagnosed using Structured Clinical Interviews for DSM-III-R patients.

Craniofacial dysmorphology refers to alternations of 'normal' or typical morphology that can be observed and/ or measured in a population.

\section{Data processing and analysis}

Data were entered via Epi data version 3.1 and analyzed using SPSS version 24. Descriptive statistics were analyzed and presented in tables. A one-way ANOVA was conducted to compare the means of craniofacial measurements among schizophrenics, their 1st-degree relatives, and healthy control. Normality checks using Levene's test were carried out for the assumption of one-way ANOVA, and the data violated the homogeneity of variances. Therefore, a Welch ANOVA and alternative post-hoc tests (i.e., a Games-Howell test instead of a Tukey post-hoc test) were conducted. Significance was set at a p-value of $\alpha=0.05$. As a result of the bulk of available evidence, consideration of a reverse timeline read-back analysis was conducted in the paper's discussion section.

\section{Ethics approval and consent to participate}

Written informed consent was obtained from the participants or their families' patients, and the study was conducted as per the Declaration of Helsinki. The ethical clearance was taken from Debre Markos University, school of medicine Ethical review committee. A permission letter has also been accepted by the hospital. 


\section{Results}

Sociodemographic characteristics and clinical details of the study samples

A total of 570 study samples, male 375(65.8\%), and female 195 (34.2\%) were included in this study. The mean ages of people with schizophrenia, their 1st-degree relatives, and healthy controls were 35.62 years, 39.97 years, and 33.56 years respectively, with no significant difference between patients and control. 44.73\% (85) of schizophrenic patients were females. $93.2 \%$ of schizophrenic patients experienced early-onset schizophrenia. $58.4 \%$ of schizophrenic patients had a history of illness for not more than 5 years.

\section{Comparison of anthropometric assessments of craniofacial} dysmorphology among study groups

The descriptive statistics showed the mean, standard deviation, and 95\% confidence intervals for the dependent variable anthropometrically assessed cranial dimensions (Table 1) and facial dimensions (Table 2) for each separate group.
A Welch ANOVA showed significant difference in mean of the head length Welch's $F(2,342.2)=8.399$, $\mathrm{p}<0.001$; head width $(\mathrm{p}<0.01)$; head height $(\mathrm{p}=0.041)$; head circumference $(\mathrm{p}=0.045)$; coronal arc length $(\mathrm{p}<0.001)$ and sagittal arc length $(\mathrm{p}<0.001)$ (Table 3$)$. Similarly, there was a significant difference in mean of the total facial height Welch's $\mathrm{F}(2,372.1)=16.33, \mathrm{p}<0.001$; the upper facial height Welch's $F(2,358.7)=22.92$, $\mathrm{p}<0.001$; the upper facial depth Welch's $\mathrm{F}$ (2, $371.6)=11.7, \mathrm{p}<0.001$; the lower facial depth Welch's $\mathrm{F}$ $(2,376.5)=12.99, \mathrm{p}<0.001)$ and the intercanthal distance Welch's $\mathrm{F}(2,370.8)=3.43, \mathrm{p}=0.033)$ between the study groups (Table 4).

The Games-Howell, multiple comparisons method, revealed that the coronal arc length and sagittal arc length among schizophrenic patients were statistically significantly longer than the healthy control $(\mathrm{p}<0.006$; $\mathrm{p}<0.001$ respectively). However, the difference between schizophrenic and healthy control regarding head circumference was marginally significant ( $\mathrm{p}=.056)$. The test also suggested the presence of a significant difference

Table 1 Descriptive statistics of anthropometric measurements of cranial dimensions among participants with schizophrenia, 1 st-degree relatives, and healthy controls

\begin{tabular}{|c|c|c|c|c|c|}
\hline \multirow{2}{*}{$\begin{array}{l}\text { Cranial anthropometric } \\
\text { measurements }(\mathrm{cm})\end{array}$} & \multirow[t]{2}{*}{$\mathbf{N}$} & \multirow[t]{2}{*}{ Mean $(\mathrm{cm})$} & \multirow[t]{2}{*}{ Std. deviation } & \multicolumn{2}{|c|}{$\mathbf{9 5 \%}$ confidence interval for mean } \\
\hline & & & & Lower bound & Upper bound \\
\hline \multicolumn{6}{|l|}{ Head length } \\
\hline Schizophrenic & 190 & 28.02 & 3.41 & 27.53 & 28.51 \\
\hline 1st degree relatives & 190 & 27.85 & 0.82 & 27.73 & 27.97 \\
\hline Healthy control & 190 & 28.2 & 0.84 & 28.08 & 28.32 \\
\hline \multicolumn{6}{|l|}{ Head width } \\
\hline Schizophrenic & 190 & 13.47 & 1.21 & 13.29 & 13.64 \\
\hline 1st degree relatives & 190 & 13.03 & 1.03 & 12.89 & 13.18 \\
\hline Healthy control & 190 & 13.43 & 0.91 & 13.3 & 13.56 \\
\hline \multicolumn{6}{|l|}{ Head height } \\
\hline Schizophrenic & 190 & 17.74 & 1.56 & 17.51 & 17.96 \\
\hline 1st degree relatives & 190 & 17.4 & 0.99 & 17.26 & 17.54 \\
\hline Healthy control & 190 & 17.44 & 1.36 & 17.24 & 17.63 \\
\hline \multicolumn{6}{|c|}{ Axial arc length (head circumference) } \\
\hline Schizophrenic & 190 & 55.25 & 4.96 & 54.54 & 55.96 \\
\hline 1st degree relatives & 190 & 55.78 & 1.65 & 55.55 & 56.02 \\
\hline Healthy control & 190 & 56.19 & 2.59 & 55.82 & 56.56 \\
\hline \multicolumn{6}{|l|}{ Coronal arc length } \\
\hline Schizophrenic & 190 & 35.67 & 2.27 & 35.34 & 35.99 \\
\hline 1st degree relatives & 190 & 34.81 & 1.97 & 34.52 & 35.09 \\
\hline Healthy control & 190 & 34.88 & 2.71 & 34.49 & 35.27 \\
\hline \multicolumn{6}{|l|}{ Sagittal arc length } \\
\hline Schizophrenic & 190 & 30.84 & 1.95 & 30.57 & 31.12 \\
\hline 1st degree relatives & 190 & 30.14 & 1.91 & 29.86 & 30.41 \\
\hline Healthy control & 190 & 29.79 & 3.09 & 29.35 & 30.24 \\
\hline
\end{tabular}


Table 2 Descriptive statistics of anthropometric measurements of facial measurements among participants with schizophrenia, 1 st-degree relatives and healthy controls

\begin{tabular}{|c|c|c|c|c|c|}
\hline \multirow{2}{*}{$\begin{array}{l}\text { Facial anthropometry } \\
\text { measurements }\end{array}$} & \multirow[t]{2}{*}{$\mathrm{N}$} & \multirow[t]{2}{*}{ Mean $(\mathrm{cm})$} & \multirow[t]{2}{*}{ Std. deviation } & \multicolumn{2}{|c|}{$95 \%$ confidence interval for mean } \\
\hline & & & & Lower bound & Upper bound \\
\hline \multicolumn{6}{|l|}{ The total facial height } \\
\hline Schizophrenic & 190 & 17.60 & 1.64 & 17.37 & 17.84 \\
\hline 1st degree relatives & 190 & 18.41 & 1.21 & 18.24 & 18.58 \\
\hline Healthy control & 190 & 18.32 & 1.22 & 18.14 & 18.49 \\
\hline \multicolumn{6}{|c|}{ Length of the upper facial region } \\
\hline Schizophrenic & 190 & 11.61 & 1.32 & 11.42 & 11.79 \\
\hline 1st degree relatives & 190 & 12.31 & .86 & 12.19 & 12.44 \\
\hline Healthy control & 190 & 12.41 & 1.39 & 12.21 & 12.61 \\
\hline \multicolumn{6}{|l|}{ Trichion to glabella } \\
\hline Schizophrenic & 190 & 3.99 & .81 & 3.87 & 4.10 \\
\hline 1st degree relatives & 190 & 4.12 & 0.52 & 4.04 & 4.19 \\
\hline Healthy control & 190 & 4.36 & 0.8 & 4.26 & 4.47 \\
\hline \multicolumn{6}{|l|}{ Glabella-subnasale } \\
\hline Schizophrenic & 190 & 7.68 & 1.27 & 7.50 & 7.86 \\
\hline 1st degree relatives & 190 & 8.28 & 1.18 & 8.11 & 8.45 \\
\hline Healthy control & 190 & 8.29 & 1.13 & 8.13 & 8.46 \\
\hline \multicolumn{6}{|l|}{ Glabella-stomion } \\
\hline Schizophrenic & 190 & 9.90 & 1.55 & 9.67 & 10.12 \\
\hline 1st degree relatives & 190 & 10.61 & 1.14 & 10.45 & 10.78 \\
\hline Healthy control & 190 & 10.42 & 1.05 & 10.27 & 10.57 \\
\hline \multicolumn{6}{|l|}{ Glabella-gnathion } \\
\hline Schizophrenic & 190 & 13.67 & & & \\
\hline 1st degree relatives & 190 & 14.31 & 1.54 & 13.45 & 13.89 \\
\hline Healthy control & 190 & 14.14 & 0.99 & 14.17 & 14.45 \\
\hline \multicolumn{6}{|l|}{ Gnathion-nasion } \\
\hline Schizophrenic & 190 & 10.79 & 1.0 & 14.0 & 14.28 \\
\hline 1st degree relatives & 190 & 11.39 & 1.43 & 10.59 & 11.0 \\
\hline Healthy control & 190 & 11.27 & 1.18 & 11.22 & 11.56 \\
\hline \multicolumn{6}{|l|}{ The lower facial region } \\
\hline Schizophrenic & 190 & 6.06 & 1.11 & 11.11 & 11.43 \\
\hline 1st degree relatives & 190 & 6.064 & 1.1 & 5.90 & 6.21 \\
\hline Healthy control & 190 & 6.10 & 0.84 & 5.94 & 6.18 \\
\hline \multicolumn{6}{|c|}{ Tragus to subnasale (middle facial depth) } \\
\hline Schizophrenic & 190 & 12.41 & 1.13 & 5.94 & 6.26 \\
\hline 1st degree relatives & 190 & 12.5 & 0.88 & 12.29 & 12.54 \\
\hline Healthy control & 190 & 12.61 & 0.7 & 12.4 & 12.60 \\
\hline \multicolumn{6}{|c|}{ Tragus to gnathion (lower facial depth) } \\
\hline Schizophrenic & 190 & 13.61 & 0.7 & 12.51 & 12.71 \\
\hline 1st degree relatives & 190 & 13.67 & 0.81 & 13.50 & 13.73 \\
\hline Healthy control & 190 & 13.97 & 0.75 & 13.56 & 13.78 \\
\hline \multicolumn{6}{|c|}{ Tragus to trichion (upper facial depth) } \\
\hline Schizophrenic & 190 & 13.67 & 0.69 & 13.87 & 14.07 \\
\hline 1st degree relatives & 190 & 13.85 & 1 & 13.53 & 13.81 \\
\hline Healthy control & 190 & 14.15 & 0.76 & 13.74 & 13.96 \\
\hline \multicolumn{6}{|l|}{ Intercanthal distance } \\
\hline Schizophrenic & 190 & 3.042 & 0.98 & 14.01 & 14.29 \\
\hline 1st degree relatives & 190 & 3.122 & 0.36 & 2.99 & 3.09 \\
\hline
\end{tabular}


Table 2 (continued)

\begin{tabular}{lllll}
\hline $\begin{array}{l}\text { Facial anthropometry } \\
\text { measurements }\end{array}$ & $\mathbf{N}$ & Mean $(\mathrm{cm})$ & Std. deviation & \multicolumn{2}{c}{$\mathbf{9 5 \% \text { confidence interval for mean }}$} \\
\hline Healthy control & & & Lower bound & Upper bound \\
\hline
\end{tabular}

Table 3 Robust tests of equality of means of cranial dimensions among study groups

\begin{tabular}{lrrrrr}
\hline $\begin{array}{l}\text { Cranial dimensions in } \\
\text { centimetre }\end{array}$ & & Statistic $^{\mathbf{a}}$ & df1 & df2 & Sig. \\
\hline Head length & Welch & 8.399 & 2 & 342.209 & .000 \\
Head width & Welch & 10.213 & 2 & 373.176 & 0.000 \\
Head height & Welch & 3.220 & 2 & 363.324 & 0.041 \\
head circumference & Welch & 3.137 & 2 & 333.502 & 0.045 \\
Coronal arc length & Welch & 8.672 & 2 & 372.092 & 0.000 \\
Sagittal arc length & Welch & 10.311 & 2 & 366.011 & 0.000 \\
\hline
\end{tabular}

${ }^{a}$ Asymptotically F distributed

Table 4 Robust tests of equality of means of facial dimensions among study groups

\begin{tabular}{llllll}
\hline Facial dimensions & & Statistic $^{\mathbf{a}}$ & df1 & df2 & Sig. \\
\hline The total facial height & Welch & 16.330 & 2 & 372.055 & 0.000 \\
$\begin{array}{l}\text { Length of the upper facial } \\
\text { region }\end{array}$ & Welch & 22.923 & 2 & 358.745 & 0.000 \\
Trichion to glabella & Welch & 10.794 & 2 & 360.153 & 0.000 \\
Glabella-subnasale & Welch & 15.245 & 2 & 377.238 & 0.000 \\
Glabella-stomion & Welch & 13.336 & 2 & 370.037 & 0.000 \\
Glabella-gnathion & Welch & 11.476 & 2 & 367.300 & 0.000 \\
Gnathion-nasion & Welch & 10.512 & 2 & 374.061 & 0.000 \\
The lower facial region & Welch & 0.091 & 2 & 370.401 & 0.913 \\
Tragus to subnasale & Welch & 2.983 & 2 & 374.245 & 0.052 \\
Tragus to gnathion & Welch & 12.992 & 2 & 376.517 & 0.000 \\
Tragus to trichion & Welch & 11.670 & 2 & 371.639 & 0.000 \\
Intercanthal distance & Welch & 3.433 & 2 & 370.791 & 0.033 \\
\hline
\end{tabular}

${ }^{a}$ Asymptotically $\mathrm{F}$ distributed

between schizophrenic patients and their 1st-degree relatives regarding head width $(\mathrm{p}=0.001)$, head height $(\mathrm{p}=0.036)$, coronal arc length $(\mathrm{p}<0.001)$, and sagittal arc length $(\mathrm{p}=0.001)$ (Table 5$)$. In the same way, schizophrenic patients had a significantly shorter total facial height $(p<0.001)$ and upper facial height $(p<0.001)$ than healthy controls. Regarding facial depth, schizophrenic patients had significantly shallow upper facial depth $(\mathrm{p}<0.001)$, middle facial depth $(\mathrm{p}=.046)$, and lower facial depth $(\mathrm{p}<0.001)$ than healthy controls (Table 6).

\section{Discussion}

Embryologically inferred measures of craniofacial dysmorphology in psychiatric diseases like schizophrenia may give an understanding of their fundamental etiologies and pathogenesis. Besides, based on destiny outline correspondences, the pattern of anomalies inside embryonic primordia may predict specific parts of brain maldevelopment, which can be assessed in brain imaging studies [20]. One approach to studying neurodevelopmental disturbances among schizophrenic patients is somatic physical evidence or neurodevelopmental markers or lingering fossil marks. These markers are measurable in adults and reflect abnormal neurodevelopmental processes that occurred before or shortly after birth [16, 17]. This anthropometric approach to the evaluation of dysmorphogenesis is reliable in the accurate assessment of 3-dimensional structures such as the craniofacial region.

In this study, patients with schizophrenia show multiple dysmorphic features of the craniofacial region that readily distinguish them from healthy controls and relatives. The Games-Howell test regarding cranial anthropometric dimensions showed that the coronal arc length and sagittal arc length among schizophrenic patients were statistically significantly longer than the healthy control. However, the difference between schizophrenic and healthy control regarding head circumference was marginally significant.

Similarly, research on morphometric characteristics of craniofacial features in patients with schizophrenia showed significantly increased coronal and Sagittal arc lengths among patients [22]. An increase in the length of the coronal arc in schizophrenia patients in our study represent a wider skull base, which may be a result of alteration in the ossification sequences of the chondrocranium and associated with the expansion of middle cranial fossa (basicranium), and consequently the volume of cerebral temporal lobe [23-25]. Regarding head circumference, our results are consistent with previous reports suggesting cranial size is increased [26] or at least is not decreased in schizophrenia $[27,28]$.

In the initial stages of embryogenesis, during the 5-13th weeks of gestation, there is a very close relationship between the craniofacial development and the 
Table 5 The Games-Howell multiple comparisons table to show specific group differences between schizophrenic patients, 1st-degree relatives, and healthy control

\begin{tabular}{|c|c|c|c|c|c|c|}
\hline \multirow{2}{*}{$\begin{array}{l}\text { Cranial } \\
\text { anthropometric } \\
\text { dimensions in }(\mathrm{cm})\end{array}$} & \multirow{2}{*}{$\begin{array}{l}\text { (I) patient control } \\
\text { status of the study } \\
\text { sample }\end{array}$} & \multirow{2}{*}{$\begin{array}{l}\text { (J) patient control } \\
\text { status of the study } \\
\text { sample }\end{array}$} & \multirow[t]{2}{*}{ Mean difference $(I-J)$} & \multirow[t]{2}{*}{ Sig. } & \multicolumn{2}{|c|}{ 95\% Confidence interval } \\
\hline & & & & & Lower bound & Upper bound \\
\hline \multirow[t]{2}{*}{ Head length } & \multirow[t]{2}{*}{ Schizophrenic } & 1st degree relatives & 0.16947 & 0.784 & -0.4315 & 0.7704 \\
\hline & & Healthy control & -0.17921 & 0.762 & -0.7808 & 0.4224 \\
\hline \multirow[t]{2}{*}{ Head width } & \multirow[t]{2}{*}{ Schizophrenic } & 1st degree relatives & $0.43474^{\mathrm{a}}$ & 0.001 & 0.1633 & 0.7062 \\
\hline & & Healthy control & 0.03526 & 0.945 & -0.2240 & 0.2945 \\
\hline \multirow[t]{2}{*}{ Head height } & \multirow[t]{2}{*}{ Schizophrenic } & 1st degree relatives & $0.33316^{\mathrm{a}}$ & 0.036 & 0.0174 & 0.6489 \\
\hline & & Healthy control & 0.29921 & 0.116 & -0.0542 & 0.6526 \\
\hline \multirow[t]{2}{*}{ Head circumference } & \multirow[t]{2}{*}{ Schizophrenic } & 1st degree relatives & -0.53474 & 0.338 & -1.4296 & 0.3601 \\
\hline & & Healthy control & -0.93737 & 0.056 & -1.8942 & 0.0195 \\
\hline \multirow[t]{2}{*}{ Coronal arc length } & \multirow[t]{2}{*}{ Schizophrenic } & 1st degree relatives & $0.86105^{\mathrm{a}}$ & 0.000 & 0.3478 & 1.3743 \\
\hline & & Healthy control & $0.79000^{\mathrm{a}}$ & 0.006 & 0.1863 & 1.3937 \\
\hline \multirow[t]{2}{*}{ Sagittal arc length } & \multirow[t]{2}{*}{ Schizophrenic } & 1st degree relatives & $0.70632^{\mathrm{a}}$ & 0.001 & 0.2404 & 1.1722 \\
\hline & & Healthy control & $1.05053^{\mathrm{a}}$ & 0.000 & 0.4262 & 1.6749 \\
\hline
\end{tabular}

${ }^{a}$ The mean difference is significant at the 0.05 level

Bold values indicates significant craniofacial differences between schizophrenic patients and healthy controls

Table 6 The Games-Howell multiple comparisons table to show specific group differences of facial dimensions between schizophrenic patients, 1st-degree relatives, and healthy controls

\begin{tabular}{|c|c|c|c|c|c|}
\hline Facial anthropometric dimensions in $(\mathrm{cm})$ & $\begin{array}{l}\text { (I) patient control } \\
\text { status of the study } \\
\text { sample }\end{array}$ & $\begin{array}{l}\text { (J) patient control } \\
\text { status of the study } \\
\text { sample }\end{array}$ & Mean difference (I-J) & Std. error & Sig. \\
\hline \multirow[t]{2}{*}{ The total facial height } & \multirow[t]{2}{*}{ Schizophrenic } & 1st degree relatives & $-0.80947^{a}$ & 0.14804 & 0.000 \\
\hline & & Healthy control & $-0.71579^{a}$ & 0.14854 & 0.000 \\
\hline \multirow[t]{2}{*}{ Length of the upper facial region } & \multirow[t]{2}{*}{ Schizophrenic } & 1st degree relatives & $-0.70789^{a}$ & 0.11420 & 0.000 \\
\hline & & Healthy control & $-0.80684^{\mathrm{a}}$ & 0.13896 & 0.000 \\
\hline \multirow[t]{2}{*}{ Trichion to glabella } & \multirow[t]{2}{*}{ Schizophrenic } & 1st degree relatives & -0.12947 & 0.06950 & 0.151 \\
\hline & & Healthy control & $-0.37211^{\mathrm{a}}$ & 0.08215 & 0.000 \\
\hline \multirow[t]{2}{*}{ Glabella-subnasale } & \multirow[t]{2}{*}{ Schizophrenic } & 1st degree relatives & $-0.59632^{\mathrm{a}}$ & 0.12556 & 0.000 \\
\hline & & Healthy control & $-0.61158^{\mathrm{a}}$ & 0.12334 & 0.000 \\
\hline \multirow[t]{2}{*}{ Glabella-stomion } & \multirow[t]{2}{*}{ Schizophrenic } & 1st degree relatives & $-0.71737^{a}$ & 0.13970 & 0.000 \\
\hline & & Healthy control & $-0.52421^{\mathrm{a}}$ & 0.13590 & 0.000 \\
\hline \multirow[t]{2}{*}{ Glabella-gnathion } & \multirow[t]{2}{*}{ Schizophrenic } & 1st degree relatives & $-0.63737^{a}$ & 0.13303 & 0.000 \\
\hline & & Healthy control & $-0.46895^{*}$ & 0.13321 & 0.001 \\
\hline \multirow[t]{2}{*}{ Gnathion-nasion } & \multirow[t]{2}{*}{ Schizophrenic } & 1st degree relatives & $-0.59789^{a}$ & 0.13438 & 0.000 \\
\hline & & Healthy control & $-0.47368^{a}$ & 0.13118 & 0.001 \\
\hline \multirow[t]{2}{*}{ The lower facial region } & \multirow[t]{2}{*}{ Schizophrenic } & 1st degree relatives & -0.00895 & 0.10036 & 0.996 \\
\hline & & Healthy control & -0.04526 & 0.11430 & 0.917 \\
\hline \multirow[t]{2}{*}{ Tragus to subnasale } & \multirow[t]{2}{*}{ Schizophrenic } & 1st degree relatives & -0.08474 & 0.08192 & 0.556 \\
\hline & & Healthy control & $-0.19474^{a}$ & 0.08171 & 0.046 \\
\hline \multirow[t]{2}{*}{ Tragus to gnathion } & \multirow[t]{2}{*}{ Schizophrenic } & 1st degree relatives & -0.05842 & 0.08000 & 0.746 \\
\hline & & Healthy control & $-0.35579^{a}$ & 0.07737 & 0.000 \\
\hline \multirow[t]{2}{*}{ Tragus to trichion } & \multirow[t]{2}{*}{ Schizophrenic } & 1st degree relatives & -0.18411 & 0.09102 & 0.108 \\
\hline & & Healthy control & $-0.48326^{a}$ & 0.10141 & 0.000 \\
\hline \multirow[t]{2}{*}{ Intercanthal distance } & \multirow[t]{2}{*}{ Schizophrenic } & 1st degree relatives & $-0.08053^{\mathrm{a}}$ & 0.03222 & 0.034 \\
\hline & & Healthy control & -0.03263 & 0.03423 & 0.607 \\
\hline
\end{tabular}

${ }^{a}$ The mean difference is significant at the 0.05 level

Bold values indicates significant craniofacial differences between schizophrenic patients and healthy controls 
ventral portion of the brain; therefore, maldevelopment in one predisposes the other for malformation $[9,29]$. In support of this, the anthropometric analysis showed the presence of maldevelopment of the middle portion of the skull in patients with schizophrenia $[9,10,30]$.

Regarding the facial measurements, schizophrenic patients in our study had a significantly shorter total facial height and upper facial height than healthy controls. They also had significantly shallow upper, middle, and lower facial depth. Another research using simple linear anthropometric measurements made with measuring tapes and calipers has shown that schizophrenic patients have more craniofacial disproportionality than healthy control subjects $[11,25,31]$.

Recent research has shown that middle cranial fossa size and shape influence the relationship between skull base shape and facial measures. The dimensions of the middle cranial fossa from which the midface attaches and grows forward from the middle cranial fossae are intimately linked to the width of the skull [32-34]. Variations in size and shape of middle cranial fossae may have predictable quantitative effects on many of the craniofacial measures that were found to differentiate those with schizophrenic from controls.

The shape and volume of the endocranial fossa are strongly correlated with the shape and volume of the adjacent parts of the brain which is lodged within the fossae. The middle cranial fossa is closely related to the anterior portion of the temporal lobe $[35,36]$. Systematic reviews and meta-analyses of published articles regarding regional brain volume have identified a decreased temporal lobe volumes among schizophrenic patients as compared with their controls [37, 38].

We predict that the anthropometric differences found in the craniofacial region of schizophrenic patients may be as a result of features related to the development of the brain/cranial fossa complex. This can be supported by the presence of significant craniofacial dysmorphology differences between schizophrenics and their first-degree relatives in this study.

A clear understanding of the relation between craniofacial dysmorphology and the risk of schizophrenia and more detailed knowledge of how the developing brain interacts with the neuro basicranial complex may help discover novel candidate exposures and genes related to schizophrenia. Research on how the brain and skull interact during development may provide new ways the understanding schizophrenia.

The consistency of results across multiple studies with ethnic and geographical backgrounds including ours supports the hypothesis that individuals with schizophrenia have increased rates of prenatal developmental disturbances than the controls and their first degree realtives.
An assessment of craniofacial dysmorphology in the population could be used as a tool among diagnostic criteria in making an early screening of schizophrenia. Similarly, it can be incorporated to diagnose schizophrenia objectively an addition to subjective analysis.

\section{Limitation of the study}

The absence of imaging modalities like MRI and CT scan was a drawback of the study, and we tried to solve it by read-back analysis.

\section{Conclusions}

The Games-Howell test revealed that the coronal arc length and sagittal arc length among schizophrenic patients were statistically significantly longer than the healthy control. However, the difference between schizophrenic and healthy control regarding head circumference was marginally significant. Schizophrenic patients had a significantly shorter total facial height and upper facial height than healthy controls. Regarding facial depth, schizophrenic patients had significantly shallow upper, middle, and lower facial depth. This finding gives clues to the neurodevelopmental basis of schizophrenia and could be used as a tool among diagnostic criteria in making an early diagnosis of schizophrenia.

\section{Supplementary Information}

The online version contains supplementary material available at https://doi. org/10.1186/s12868-021-00663-y.

Additional file 1. Landmarks on craniofacial region used for anthropometric assessments.

\section{Acknowledgements}

We would like to say thank you to Debre Markos university's research directorate that supports data collection. Debre Markos comprehensive specialized hospital, staff of psychiatry department, and Debre Markos university Medical interns thank you very much for your continuous support of the data collection procedure.

\section{Authors' contributions}

BT and GS conceived and designed the study. BT wrote the initial draft of the manuscript, which was critically reviewed by GS and had analyzed data. All authors read and approved the final manuscript.

Funding

No funding.

Availability of data and materials

The dataset supporting the conclusion of this article is available from the authors on request. 


\section{Declarations}

\section{Ethics approval and consent to participate}

Written informed consent was obtained from the participants or their families, and the study was conducted as per the Declaration of Helsinki. The ethical clearance was taken from Debre Markos University, school of medicine Ethical review committee. The hospital has also accepted a permission letter.

\section{Consent for publication}

Not applicable.

\section{Competing interests}

The authors declare that they have no competing interests.

\section{Author details}

'Department of Biomedical Sciences, Debre Markos University, Debre Markos, Ethiopia. ${ }^{2}$ Department of Anatomy, Addis Ababa University, Addis Ababa, Ethiopia.

Received: 11 April 2021 Accepted: 4 September 2021

Published online: 29 September 2021

\section{References}

1. Compton MT, Walker EF. Physical manifestations of neurodevelopmental disruption: are minor physical anomalies part of the syndrome of schizophrenia? Schizophr Bull. 2009:35(2):425-36

2. Waddington $J$, et al. Early cerebro-craniofacial dysmorphogenesis in schizophrenia: a lifetime trajectory model from neurodevelopmental basis to 'neuroprogressive'process. J Psychiatr Res. 1999;33(6):477-89.

3. Akbarian $\mathrm{S}$, et al. Altered distribution of nicotinamide-adenine dinucleotide phosphate-diaphorase cells in frontal lobe of schizophrenics implies disturbances of cortical development. Arch Gen Psychiatry. 1993:50(3):169-77.

4. Murray RM, Lewis SW. Is schizophrenia a neurodevelopmental disorder? Br Med J. 1987;295(6600):681.

5. Waddington JL, et al. The neurodevelopmental basis of schizophrenia: clinical clues from cerebro-craniofacial dysmorphogenesis, and the roots of a lifetime trajectory of disease. Biol Psychiat. 1999;46(1):31-9.

6. McGrath JJ, et al. The neurodevelopmental hypothesis of schizophrenia: a review of recent developments. Ann Med. 2003;35(2):86-93.

7. Woods BT. Is schizophrenia a progressive neurodevelopmental disorder? Toward a unitary pathogenetic mechanism. Am J Psychiatry. 1998;155(12):1661-70

8. Diewert VM, Lozanoff S. A morphometric analysis of human embryonic craniofacial growth in the median plane during primary palate formation. J Craniofac Genet Dev Biol. 1993:13:147-147.

9. Hennessy RJ, et al. Three-dimensional laser surface imaging and geometric morphometrics resolve frontonasal dysmorphology in schizophrenia. Biol Psychiat. 2007;61(10):1187-94.

10. Lane A, et al. The anthropometric assessment of dysmorphic features in schizophrenia as an index of its developmental origins. Psychol Med. 1997;27(5):1155-64.

11. Elizarraras-Rivas J, et al. Minor physical anomalies and anthropometric measures in schizophrenia: a pilot study from Mexico. Schizophr Res. 2003:62(3):285-7.

12. Smith DW. Recognisable patterns of human malformation. Philadelphia: WB Saunders; 1988

13. Pinsky L. Informative morphogenetic variants. In: Kalter $\mathrm{H}$, editor. Issues and reviews in teratology. Berlin: Springer; 1985. p. 135-70.

14. Krouse JP, Kauffman JM. Minor physical anomalies in exceptional children: a review and critique of research. J Abnorm Child Psychol. 1982:10(2):247-64.
15. Persaud T. Teralogenesis-experimental aspects and clinical implications. Exp Pathol. 1979;1:1-128.

16. Green MF, et al. Preliminary evidence for an association between minor physical anomalies and second trimester neurodevelopment in schizophrenia. Psychiatry Res. 1994;53(2):119-27.

17. McDonald C, Murray RM. Early and late environmental risk factors for schizophrenia. Brain Res Rev. 2000;31(2-3):130-7.

18. Pantelis $C$, et al. Early and late neurodevelopmental disturbances in schizophrenia and their functional consequences. Aust N Z J Psychiatry. 2003;37(4):399-406

19. Couly GF, Coltey PM, Le Douarin NM. The triple origin of skull in higher vertebrates: a study in quail-chick chimeras. Development. 1993;117(2):409-29.

20. Deutsch CK, et al. Skewing of the brain midline in schizophrenia. NeuroReport. 2000;11(18):3985-8.

21. Farkas LG. Anthropometry of the head and face. New York: RavenPress; 1994.

22. Fakhroddin M, Ahmad G, Imran S. Morphometric characteristics of craniofacial features in patients with schizophrenia. Afr J Psychiatry. 2014. https://doi.org/10.4172/Psychiatry.1000107.

23. Hennessy RJ, et al. Three-dimensional laser surface imaging and geometric morphometrics resolve frontonasal dysmorphology in schizophrenia. Biol Psychiatry. 2007;61(10):1187-94

24. Donovan-Lepore A-M, et al. Quantitative craniofacial anomalies in a racially mixed schizophrenia sample. Biol Psychiatry. 2006;59(4):349-53.

25. McGrath J, et al. Minor physical anomalies and quantitative measures of the head and face in patients with psychosis. Arch Gen Psychiatry. 2002;59(5):458-64.

26. Weinberger DR, et al. Hat size in schizophrenia. Arch Gen Psychiatry. 1987:44(7):672-672

27. Andreasen NC, et al. Magnetic resonance imaging of the brain in schizophrenia: the pathophysiologic significance of structural abnormalities. Arch Gen Psychiatry. 1990;47(1):35-44.

28. Breier A, et al. Brain morphology and schizophrenia: a magnetic resonance imaging study of limbic, prefrontal cortex, and caudate structures. Arch Gen Psychiatry. 1992;49(12):921-6.

29. Marcucio RS, et al. Molecular interactions coordinating the development of the forebrain and face. Dev Biol. 2005;284(1):48-61.

30. Hennessy RJ, Kinsella A, Waddington JL. 3D laser surface scanning and geometric morphometric analysis of craniofacial shape as an index of cerebro-craniofacial morphogenesis: initial application to sexual dimorphism. Biol Psychiatry. 2002:51(6):507-14.

31. MiShrA V, et al. Anthropometerical association of the craniofacial dysmorphology with schizophrenia. J Clin Diagn Res . 2012;6(10):1620.

32. Enlow DH. Facial growth. Philadelphia:WB Saunders Company; 1990.

33. Lieberman DE, Pearson OM, Mowbray KM. Basicranial influence on overall cranial shape. J Hum Evol. 2000;38(2):291-315.

34. Sperber GH, Sperber SM. Craniofacial embryogenetics and development, third. Br Dent J. 2019;227(9):773.

35. Moss MLJCAG, NTMP. Development from a surgical perspective. New York. Growth and development of the craniofacial complex: an epigenetic viewpoint. 1995; p. 1-7.

36. Hadziselimović $H$, Cus M. The appearance of internal structures of the brain in relation to configuration of the human skull. Acta Anat (Basel). 1966;63(3):289-99.

37. McCarley RW, et al. MRI anatomy of schizophrenia. Biol Psychiatry. 1999:45(9):1099-119.

38. Wright IC, et al. Meta-analysis of regional brain volumes in schizophrenia. Am J Psychiatry. 2000;157(1):16-25.

\section{Publisher's Note}

Springer Nature remains neutral with regard to jurisdictional claims in published maps and institutional affiliations. 